\title{
Respiratory Muscle Strength Predicts Decline in Mobility in Older Persons
}

\author{
A.S. Buchman ${ }^{a, b}$ P.A. Boyle ${ }^{a, c}$ R.S. Wilson ${ }^{a-c}$ S. Leurgans ${ }^{a, b}$ R.C. Shah ${ }^{a, d}$ \\ D.A. Bennett ${ }^{a}, b$ \\ ${ }^{a}$ Rush Alzheimer's Disease Center and Departments of ${ }^{\mathrm{b}}$ Neurological Sciences, ${ }^{\mathrm{c} B e h a v i o r a l ~ S c i e n c e s ~ a n d ~}$ \\ dFamily Practice, Rush University Medical Center, Chicago, III., USA
}

\section{Key Words}

Respiratory muscle strength • Mobility • Lower extremity strength • Physical activity • Aging

\begin{abstract}
Objectives: To test the hypothesis that respiratory muscle strength is associated with the rate of change in mobility even after controlling for leg strength and physical activity. Methods: Prospective study of 890 ambulatory older persons without dementia who underwent annual clinical evaluations to examine change in the rate of mobility over time. Results: In a linear mixed-effect model adjusted for age, sex, and education, mobility declined about 0.12 unit/year, and higher levels of respiratory muscle strength were associated with a slower rate of mobility decline (estimate 0.043 , SE $0.012, p<0.001)$. Respiratory muscle strength remained associated with the rate of change in mobility even after controlling for lower extremity strength (estimate 0.036 , SE $0.012, p=0.004)$. In a model that included terms for respiratory muscle strength, lower extremity strength and physical activity together, all three were independent predictors of mobility decline in older persons. These associations remained significant even after controlling for body composition, global cognition, the development of dementia, parkinsonian signs, possible pulmonary disease, smoking, joint pain and chronic diseases. Conclusion: Respiratory muscle strength is associated with mobility decline in older persons
\end{abstract}

\begin{tabular}{ll}
\hline KARGER & ( ) 2008 S. Karger AG, Basel \\
Fax +41613061234 & \\
$\begin{array}{l}\text { E-Mail karger@karger.ch } \\
\text { www.karger.com }\end{array}$ & $\begin{array}{l}\text { Accessible online at: } \\
\text { www.karger.com/ned }\end{array}$
\end{tabular}

independent of lower extremity strength and physical activity. Clinical interventions to improve respiratory muscle strength may decrease the burden of mobility impairment in the elderly.

Copyright $\odot 2008$ S. Karger AG, Basel

\section{Introduction}

Loss of muscle strength and increasing mobility impairment are common with age and are associated with significant morbidity and mortality [1-4]. Thus efforts to intervene and modify the course of age-related motor decline are a growing public health concern in our aging population. Physical activity is well known to have beneficial effects on mobility, and it is widely thought that one of the ways in which physical activity may benefit mobility in the elderly is through its effects on lower extremity muscle structure and function. In a previous study, we showed that physical activity and lower extremity strength are independent predictors of mobility decline in older persons [5]. Thus, although increased physical activity may improve lower extremity strength, the beneficial effect of physical activity on mobility is likely to involve other pathways. Although respiratory muscle strength declines with increasing age, little is known about its association with the rate of change in mobility in older persons without respiratory complaints. Further,

Aron S. Buchman, MD

Rush Alzheimer's Disease Center, Rush University Medical Center Armour Academic Facility, Suite 1038, 600 South Paulina Street

Chicago, IL 60612 (USA)

Tel. +1 312942 8942, Fax +1 312563 4604, E-Mail Aron_S_Buchman@rush.edu 
Fig. 1. Participants eligible for current analyses. Total number of participants who were enrolled in the Memory and Aging Project is shown and those who were excluded because they did not meet the inclusion criteria for these analyses.

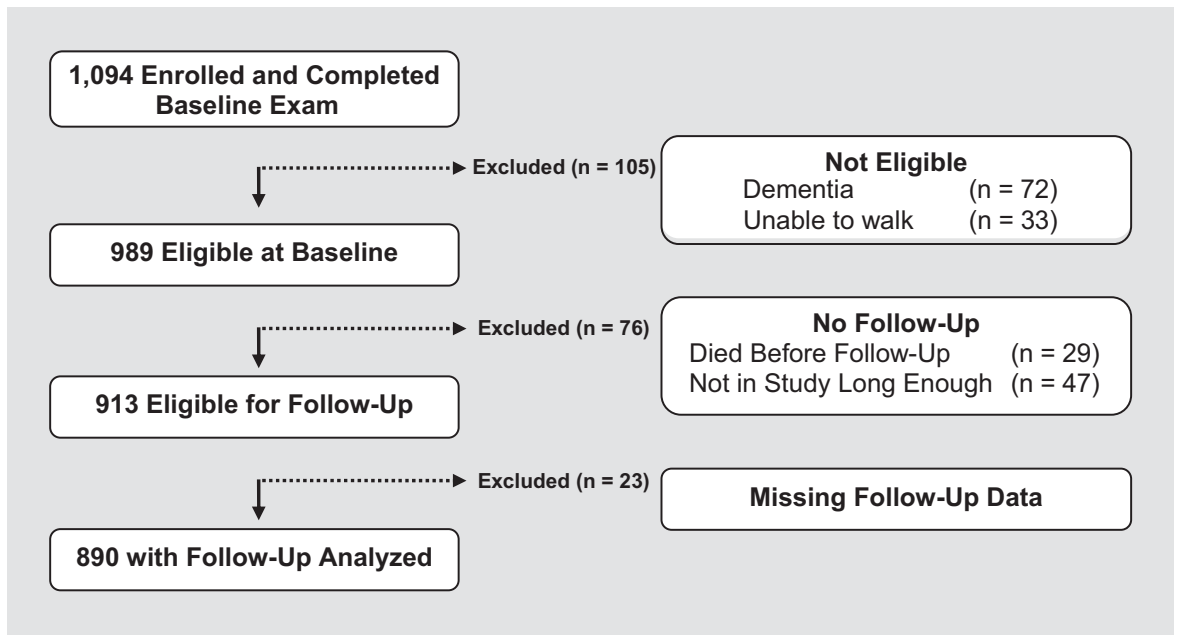

it is not known whether respiratory muscle strength is associated with mobility after controlling for lower extremity muscle strength and physical activity $[6,7]$.

We used data from nearly 900 community-based ambulatory older persons without dementia who were participating in the Rush Memory and Aging Project and had undergone annual clinical assessments to investigate the extent to which respiratory muscle strength is associated with the rate of change in mobility after controlling for lower extremity strength and physical activity [8]. We used linear mixed-effect models to examine the associations of respiratory muscle strength with the rate of change in mobility when controlling for lower extremity strength. Next, we examined whether respiratory muscle strength was still associated with the rate of change in mobility after controlling for both physical activity and lower extremity strength in a single model. Finally, we repeated these analyses controlling for several covariates that might affect the primary associations.

\section{Methods}

Participants

All participants were from the Rush Memory and Aging Project, a community-based, longitudinal clinical-pathologic investigation of chronic conditions of old age. Participants were recruited from more than 40 residential facilities across the metropolitan Chicago area, including subsidized senior housing facilities, retirement communities, and retirement homes, in addition to social service agencies and church groups. Participants agreed to annual detailed clinical evaluations (described below) and all evaluations were performed at the parent facility or the participants' homes to reduce burden and enhance follow-up participation [8]. The study was conducted in accordance with the latest version of the Declaration of Helsinki and was approved by the Institutional Review Board of Rush University Medical Center.

The Memory and Aging Project began in 1997 and the overall follow-up rate is about $95 \%$ of survivors. Because of the rolling admission and mortality, the length of follow-up and number of examinations varies across participants. Further, because the collection of data on respiratory function was not added until 2001, it was only available on a subset of Memory and Aging Project participants. To maintain the temporal relation between respiratory muscle strength and mobility, we included the first respiratory muscle strength test as the predictor and considered the mobility measure obtained at that evaluation the 'baseline' for this study; all subsequent mobility measures available for each participant were used to calculate the rate of change in mobility. There were four requirements for inclusion in these analyses: (1) the absence of dementia at the baseline evaluation; (2) the ability to ambulate at baseline; (3) a valid respiratory muscle strength testing at baseline and (4) one or more valid follow-up mobility scores.

At the time of these analyses, 1,094 participants had enrolled and completed a baseline evaluation; of these 105 were excluded because of dementia or inability to ambulate at baseline and 76 were excluded since they were not eligible for follow-up exam. Of 913 who were eligible for follow-up exam 23 had missing follow data (participation rate of $>97 \%$ ), leaving 890 for these analyses (fig. 1). Their mean age at baseline was 80.6 years $(S D=7.22)$, mean education was 14.6 years $(S D=2.9)$, mean Mini-Mental State Examination score was $28.0(\mathrm{SD}=2.08$; median $=28$; range $18-30)$, BMI was 27.3 ( $\mathrm{SD}=5.23$; median $=26$; range $17-63$ ) and $75.6 \%$ were women. Following the baseline exam, participants were examined annually [average interval between annual follow-up evaluations was 11.9 months $(\mathrm{SD}=2.0$ months)] with a mean follow-up of 3.0 years $(\mathrm{SD}=1.3)$. Thus, on average, there were 4 mobility assessments for each participant $(\mathrm{SD}=1.2$; range 2-6) used for these longitudinal analyses.

Subjects underwent a uniform structured clinical evaluation including a medical history, neurologic examination, and cognitive performance testing. Details of the clinical evaluation have been previously described [8]. A battery of 21 cognitive tests was administered at each evaluation to assess cognitive function. The Mini- 
Mental State Examination was used to describe the cohort [9]. Scores on 19 tests were used to create a composite measure of global cognitive function. To compute the composite measure of global cognitive function, raw scores on each of the individual tests were converted to $\mathrm{z}$ scores using the baseline mean and standard deviation of the entire cohort, and the $\mathrm{z}$ scores of all 19 tests were averaged. Psychometric information about this composite measure of global cognition is contained in previous publications [10].

Dementia was diagnosed in a three-step process. First, a computer scored the neuropsychological tests and applied an education-adjusted impairment rating for 11 tests commonly used for the clinical classification of Alzheimer's disease [11]. Next, following a review of the results of all cognitive tests, an experienced, board-certified clinical neuropsychologist rendered a clinical judgment regarding the presence of cognitive impairment. Finally, all participants were evaluated in person by a physician with expertise in the evaluation of older persons with and without dementia. The physician determined the presence of dementia (when appropriate) based on the in-person evaluation and a review of all available clinical data and their examination. The diagnosis of dementia followed the criteria of the joint working group of the National Institute of Neurological and Communicative Disorders and Stroke and the Alzheimer's Disease and Related Disorders Association. These require a history of cognitive decline and evidence of impairment in two or more domains of cognition, one of which must be memory, for classification as Alzheimer's disease [12].

\section{Mobility}

We asked people to walk eight feet and turn $360^{\circ}$ and measured time and number of steps on each task. Those unable to perform a task were given a score of 0 ; for the remainder, scores on each measure were divided into quintiles: scores of 5 were assigned to the quintile with the fastest time and fewest steps and scores of 1 to the quintile with the slowest times and most steps. We used components of variance analysis to examine the variability of the components used to construct composite mobility. Composite mobility was collected annually and each of its four subcomponents was based on two trials. We considered three sources that could contribute to the variation of composite mobility: (1) within subject, (2) visit within subject and (3) trial within visit. When considering each of the four components, on average, the variation due to subject was $31.5 \%$ of the total variation; visit within subject was $39.9 \%$ and trial within visit contributed $28.7 \%$ of the total variation. The variation of the trial within visit is further reduced since the two trials which were collected are averaged together to yield each of the four subcomponents. Since averaging trials reduces an already small source of variation, we have not employed other methods to compensate for variation within visit. Each of the four component measures were converted to $\mathrm{z}$ scores and averaged to yield composite mobility as previously described [5].

\section{Respiratory Muscle Strength}

Respiratory muscle strength was based on measures of maximal inspiratory (MIP) and expiratory (MEP) pressures [13, 14]. A hand-held device that contains a pressure-sensitive transducer was used to assess MIP (in $\mathrm{cm} \mathrm{H}_{2} \mathrm{O}$ ) and MEP (in $\mathrm{cm} \mathrm{H}_{2} \mathrm{O}$;) MicroMouth Pressure Meter MP01; MicroMedical Ltd., Kent, UK). Participants were requested take a deep breath and to seal their lips securely around the mouthpiece of this portable pressure meter. The subjects were verbally encouraged to maximally inspire for MIP or maximally expire for MEP and to sustain their maximal level for at least $1 \mathrm{~s}$. Two trials of both MIPs and MEPs were collected at baseline. The mean scores for MIPs and MEPs were converted to $\mathrm{z}$ scores, using the mean and standard deviation of all study participants at baseline. Both z scores were averaged to yield a composite measure of respiratory muscle strength [15]. We used components of variance analysis to examine the contribution of trial to trial variation and subject to the subcomponents (MIP and MEP) of baseline composite respiratory muscle strength. On average, the contribution of the trial to trail variation to total variation of both subcomponents was $14.8 \%$ and was much smaller than the variation due to subject (85.2\%). The trial to trial variation is further reduced since the two trials which were collected are averaged together to yield each of the two subcomponents. Since this process reduces an already small source of variation, we have not employed other methods to compensate for variation due to trial.

\section{Lower Extremity Strength}

Hand-held dynamometers (Lafayette Manual Muscle Test System, Model 01163, Lafayette, Ind., USA) [16] were used to assess muscle strength in both lower extremities (hip flexion, knee extension, plantar flexion, and ankle dorsiflexion). The mean score for each muscle group was converted to a $\mathrm{z}$ score, using the mean and standard deviation of all study participants at baseline and the $\mathrm{z}$ scores of all the lower extremity muscles were averaged to yield lower extremity strength as previously described [5].

\section{Physical Activity}

Physical activity was assessed using questions adapted from the 1985 National Health Interview Survey [17]. Activities included walking for exercise, gardening or yard work, calisthenics or general exercise, bicycle riding, and swimming or water exercise. Minutes spent engaged in each activity were summed and expressed as hours of activity per week, as previously described [5].

\section{Other Covariates}

Gender, age, and years of education were obtained at baseline evaluation. Weight and height were measured and used to calculate BMI. Parkinsonian signs were based on a modified version of the motor portion of the Unified Parkinson's Disease Rating Scale [18]. Joint pain was based on participant report of joint pain. We summarized vascular risk factors as the number of the following risk factors: hypertension, diabetes mellitus, and smoking. Vascular disease burden was the number of four vascular diseases: myocardial infarction, congestive heart failure, claudication, and stroke, as previously described [18]. In addition, forced expiratory volume in $1 \mathrm{~s}\left(\mathrm{FEV}_{1}\right)$ and forced vital capacity (FVC) were measured with a hand-held spirometer (MicroPlus ${ }^{\circledR}$ Spirometer MS03, MicroMedical Ltd.). In order to examine the possibility that participants with possible pulmonary disease influenced the results, we considered participants to have possible pulmonary disease if the ratio of $\mathrm{FEV}_{1} / \mathrm{FVC}$ was $\leq 0.7$, as suggested by previous literature [19]. In addition, for these analyses, participants who were receiving one or more medications used to treat chronic pulmonary diseases including anticholinergics, $\beta$-adrenergics, theophylline, steroid inhalants, and leukotrienes were considered to have possible pulmonary disease. Medications were inspected and coded using the Medi-Span ${ }^{\circledR}$ system (Medi-Span, Inc.) [8]. 


\section{Analysis}

Pearson ( $r$ ) correlations were used to assess the relationship of gait measures with age and education, $t$ tests were used to compare men and women and Spearman (rho) correlations were used to assess the relationship of physical performance measures. Linear mixed-effect models were used to determine whether respiratory muscle strength predicted mobility decline over several years of follow-up. In mixed-effect models, the rate of change in mobility (slope) for an individual participant is postulated to follow the mean path of change in mobility for the group, except for random effects that may contribute variability in both mobility performance at baseline and the rate of change in mobility over time. Therefore, mixed-effect models include linear model terms for the mean person-specific intercepts (terms which appear as main effects in the mixed model), and terms for the mean person-specific slopes (terms which appear as interactions with time). The person-specific deviations from the means are modeled as random effects on both slope and intercept. This approach reduces the noise in the parameter estimates of individual slopes and yields a more precise estimate of the effects of the covariates of interest (e.g., respiratory muscle strength) on person-specific change in mobility. Linear mixed-effect models are particularly well-suited to modeling person-specific mobility based on repeated annual measures of mobility. These models are robust and have the practical advantages that individuals included need not have the same number of observations and the time between observations is not assumed to be constant within or between persons. We have used this approach in several previous longitudinal studies of mobility and cognitive function [5, 20, 21].

All models controlled for age, sex, education and their interaction with time. Then we added a pair of terms for baseline respiratory muscle strength and its interaction with time to the core model. Next, because we had previously shown that lower extremity strength and physical activity were relatively independently associated with mobility decline, we first added a pair of terms for baseline lower extremity strength and its interaction with time and then another pair of terms for baseline physical activity and its interaction with time to determine if controlling for lower extremity strength and physical activity changed the association between respiratory muscle strength and the rate of change in mobility. Finally, we examined several covariates that might affect these associations. A term for each covariate was added to the previous model as well as a term for its interaction with time. Since both low and high values of BMI can be associated with adverse health consequences, we added a term for BMI and a quadratic term for BMI $(\mathrm{BMI} \times \mathrm{BMI})$ as well as a term for their interaction with time. Model assumptions of linearity, normality, independence of errors, and homoscedasticity of errors were examined graphically and analytically and were adequately met [22]. Programming was done using SAS software (SAS Institute, Cary, N.C., USA) [23].

\section{Results}

\section{Baseline Mobility}

Baseline mobility ranged from -1.55 to 1.35 (mean $=$ $0.14, \mathrm{SD}=0.77$ ) with higher scores indicating better performance. Baseline mobility was inversely related to age $(\mathrm{r}=-0.33, \mathrm{p}<0.001)$, positively associated with education $(\mathrm{r}=0.14, \mathrm{p}<0.001)$, and was higher in men than women (mean difference $=0.21$ unit, $\mathrm{t}=-3.50, \mathrm{p}<0.001$ ).

\section{Respiratory Muscle Strength and Change in Mobility}

Baseline respiratory muscle strength ranged from -2.01 to 3.20 (mean $=0.01 ; \mathrm{SD}=0.85)$, with higher scores indicating better performance. Respiratory muscle strength was inversely related to age $(r=-0.22, \mathrm{p}<0.001)$, positively associated with education $(\mathrm{r}=0.14, \mathrm{p}<0.001)$, and men were stronger than women (mean difference $=$ 0.79 unit, $\mathrm{t}=-11.80, \mathrm{p}<0.001)$. There was a moderate association of respiratory muscle strength with lower extremity strength (rho $=0.35, \mathrm{p}<0.001)$ and a mild association with physical activity ( $\mathrm{rho}=0.12, \mathrm{p}<0.001)$. There was a weak association between lower extremity strength and physical activity (rho $=0.09, \mathrm{p}<0.01$ ).

We examined the individual differences in the rate of change in mobility using a linear mixed-effect model controlling for age, sex and education and their interaction with time. Mobility declined by about 0.16 unit/year (estimate $=-0.161 ; \mathrm{SE}=0.010, \mathrm{p}<0.001$ ). Next we added a term for baseline respiratory muscle strength and its interaction with time to the previous model to examine whether respiratory muscle strength was related to the rate of change in mobility. In this model mobility declined by about 0.122 unit/year and each 1-unit increase in respiratory strength at baseline was associated with a slowed decline in mobility of 0.043 unit/year. Therefore a person with respiratory strength 1 unit above the mean at baseline would show about a 35\% slower rate of decline in mobility (mean decline: about 0.079 unit/year, respiratory muscle strength $\times$ time: table 1 , model A). Another way of describing the association of respiratory muscle strength with mobility is to compare it to the association of age and mobility decline. There was a significant interaction between baseline age and mobility, such that for each reduced year of age, mobility decline was reduced by almost 3\% (age $\times$ time: estimate $=0.003 ; \mathrm{SE}=0.013, \mathrm{p}=$ $0.007)$. Thus, the effect of an additional unit of respiratory muscle strength at baseline on mobility decline was the same as about being 13 years younger at baseline.

Next, because we had previously shown that lower extremity strength and physical activity were relatively independently associated with mobility decline, we examined whether controlling for lower extremity strength and physical activity confounded the association of respiratory muscle strength with the rate of change in mobility. First we add a term for lower extremity strength to the previous model and the association of respiratory muscle strength 
Table 1. Respiratory muscle strength is associated with change in mobility even after controlling for lower extremity strength and physical activity

\begin{tabular}{lrrr}
\hline Term & Model A, estimate & Model B, estimate & Model C, estimate \\
\hline Time & $-0.122(0.010,<0.001)$ & $-0.117(0.010,<0.001)$ & $-0.143(0.012,<0.001)$ \\
Respiratory muscle strength & $0.211(0.031,<0.001)$ & $0.183(0.032,<0.001)$ & $0.176(0.032,<0.001)$ \\
Respiratory muscle strength $\times$ time & $0.043(0.012,<0.001)$ & $0.036(0.012,0.004)$ & $0.032(0.012,0.008)$ \\
Lower extremity strength & & $0.100(0.030,<0.001)$ & $0.095(0.029,0.001)$ \\
Lower extremity strength $\times$ time & & $0.025(0.012,0.033)$ & $0.026(0.012,0.026)$ \\
Physical activity & & & $0.019(0.007,0.003)$ \\
Physical activity $\times$ time & & $0.009(0.002,<0.001)$
\end{tabular}

Estimated from linear mixed-effect models adjusted for age, sex, education, and their interactions with time (i.e. mean annual linear change in mobility for an average participant). We sequentially added terms, first for baseline respiratory muscle strength and its interaction with time (model A); we next added terms for lower extremity strength and its interaction with time (model B), and finally, we added terms for physical activity and its interaction with time (model C). Values in parentheses represent SE followed by $\mathrm{p}$ value. and rate of change in mobility was unchanged (table 1 , model B). Next we added a term for physical activity to the previous model and the estimates for respiratory muscle strength, lower extremity strength and physical activity remained associated with mobility (table 1 , model $\mathrm{C}$ ). These results suggest that all three of these performance measures, respiratory muscle strength, lower extremity strength and physical activity, make relatively independent contributions to the rate of change in mobility.

Finally, we examined several covariates that might affect the different physical performance measures or respiratory muscle strength and might thereby affect their association with mobility including: body composition, baseline global cognition and the subsequent development of dementia during the course of the study, parkinsonian signs, comorbid conditions including possible pulmonary disease as evidenced by a ratio of $\mathrm{FEV}_{1} / \mathrm{FVC}$ $\leq 0.7$ or the use of medications used to treat pulmonary diseases, joint pain, vascular diseases and vascular risk factors, including smoking. Even after adding terms for these covariates to model C (table 1), respiratory muscle strength remained associated with mobility decline (results not shown).

\section{Discussion}

In a group of 890 ambulatory older persons without dementia at baseline and with a rate of change of mobility determined over an average of 3 years of follow-up, we found that respiratory muscle strength is associated with the rate of change in mobility. Further, when respiratory muscle strength was considered together in a single model controlling for lower extremity strength and physical activity, all terms remained relatively independently associated with the rate of change in mobility. The latter findings were robust and persisted even after controlling for a number of other covariates that may contribute to mobility including body composition, baseline cognition, subsequent development of dementia, parkinsonian signs, as well as several comorbid conditions including possible pulmonary disease, smoking or vascular conditions and risk factors. These results suggest that respiratory muscle strength is associated with loss of mobility in older adults even after controlling for lower extremity strength and physical activity. These findings suggest that interventions focused on improving respiratory muscle strength may decrease mobility impairment in the elderly.

Mobility decline in the elderly is common and associated with incident disability, institutionalization and mortality [2]. Identifying factors that predict change in mobility is important for the development of interventions to modify this growing public health concern in our aging population. Muscle strength declines with age and numerous cross-sectional studies have shown that low muscle strength is usually associated with decreased mobility $[4,6,7]$. Although most previous clinical research has focused on age-related changes in appendicular muscle strength, age-related changes in respiratory muscle strength have also been reported [13]. Respiratory muscles include the inspiratory and expiratory muscle groups. The diaphragm, internal intercostals of the parasternal region, external intercostals, and other accessory muscles 
constitute the inspiratory muscles. The lateral internal intercostals and abdominal muscles constitute the expiratory muscles. Lower respiratory muscle strength may lead to impaired pressure gradients and air exchange at the alveolar surface which could limit mobility.

Importantly, there are limited data on the association of respiratory muscle strength with mobility in older persons without known pulmonary disease [24-26]. Impaired respiratory muscle strength has been extensively studied in patients with chronic lung and cardiac disease and is associated with decreased mobility in these conditions [27-30]. Thus, the association between respiratory muscle strength and mobility observed in this study is consistent with previous studies of persons with diverse medical conditions. The current study extends previous studies and shows that respiratory muscle strength is related to mobility decline even after controlling for lower extremity strength and physical activity in older persons without known pulmonary disease. Since both respiratory and lower extremity strength are independently associated with mobility, both need to be considered when trying to explicate the biology of mobility decline.

Physical activity is associated with a wide range of important health outcomes including loss of mobility but the biology of this association is poorly understood [31]. Data from cross-sectional studies suggest that physical activity is at least partially linked with mobility through its effect on muscle function including strength or power [32]. However, there are few longitudinal studies of the relationship between physical activity, muscle strength and the rate of decline in mobility including recent work in this cohort [5]. Some clinical intervention studies that have aimed to increase muscle strength have reported improved performance, but others have failed to find beneficial effects $[33,34]$. Moreover, whereas some have shown that physical activity is associated with improved mobility via its effect on muscle and cardiovascular fitness, others have reported improved health benefits from higher levels of routine physical activity despite no demonstrable improvement in muscle function or changes in cardiac output, suggesting the possible importance of other pathways [35]. Thus, while physical activity may improve muscle structure and function, this is not the only mechanism through which physical activity has a beneficial effect on mobility. We are not aware of studies that have examined whether physical activity is associated with mobility after controlling for both respiratory and lower extremity muscle strength [36]. The current study showed that three important subsystems necessary for mobility including physical activity, respiratory and

Respiratory Muscle Strength and

Mobility lower extremity muscle strength make independent contributions to the rate of decline in mobility even after controlling for other covariates. This suggests that clinical interventions may need to focus on multiple subsystems that make independent contributions to mobility loss and suggest that there are alternative pathways through which interventions may modify mobility impairment in the elderly [37].

Our study has several limitations. First, the participants in this study are a selected group having agreed to postmortem donation, so these results will need to be replicated in the general population. Respiratory muscle strength was measured with a hand-held device in the community and in contrast to laboratory testing only two trials were collected. In addition, hand-held dynamometry was used to measure lower extremity strength, which does not measure all of the important aspects of strength. Therefore, although respiratory and lower extremity strength was both associated with mobility decline, the use of a more sensitive measure of strength might have shown attenuation of the association of physical activity with mobility.

Despite these limitations, several factors increase confidence in the findings from this study. Perhaps most importantly, strength was evaluated as part of a uniform structured clinical evaluation and incorporated many widely accepted and reliable performance measures; strength testing was done in multiple lower extremity muscles. Based on a uniform clinical evaluation and widely accepted diagnostic criteria, persons with dementia were excluded from analyses and a relatively large number of older persons were studied, so that there was adequate statistical power to identify the associations of interest while controlling for potentially confounding variables.

\section{Acknowledgments}

This work was supported by National Institute on Aging grants R01AG17917 and R01AG24480, the Illinois Department of Public Health, and the Robert C. Borwell Endowment Fund. We thank all the participants in the Rush Memory and Aging Project. We also thank Traci Colvin and Tracey Nowakowski for project coordination, Barbara Eubeler, Mary Futrell, Karen Lowe Graham and Pam A. Smith for participant recruitment, John Gibbons and Greg Klein for data management, Liping $\mathrm{Gu}$ for statistical programming and the staff of the Rush Alzheimer's Disease Center.

Neuroepidemiology 2008;31:174-180 179 


\section{References}

1 Woo J, Ho SC, Yu AL: Walking speed and stride length predicts 36 months dependency, mortality, and institutionalization in Chinese aged 70 and older. J Am Geriatr Soc 1999;47:1257-1260.

-2 Guralnik JM, Ferrucci L, Pieper CF, et al: Lower extremity function and subsequent disability: consistency across studies, predictive models, and value of gait speed alone compared with the short physical performance battery. J Gerontol A Biol Sci Med Sci 2000;55:M221-M231.

3 Cesari M, Kritchevsky SB, Penninx BWHJ, et al: Prognostic value of usual gait speed in well-functioning older people - results from the health, aging and body composition study. J Am Geriatr Soc 2005;53:1675-1680.

$\checkmark 4$ Verghese J, LeValley A, Hall CB, Katz MJ, Ambrose AF, Lipton RB: Epidemiology of gait disorders in community-residing older adults. J Am Geriatr Soc 2006;54:255-261.

$\checkmark 5$ Buchman AS, Wilson RS, Boyle PA, Tang Y, Fleischman DA, Bennett DA: Physical activity and leg strength predict decline in mobility performance in older persons. J Am Geriatr Soc 2007;55:1618-1623.

6 Roos MR, Rice CL, Vandervoort AA: Agerelated changes in motor unit function. Muscle Nerve 1997;20:679-690.

7 Vandervoort AA: Aging of the human neuromuscular system. Muscle Nerve 2002;25 $17-25$.

$\checkmark 8$ Bennett DA, Schneider JA, Buchman AS, Mendes de Leon C, Bienias JL, Wilson RS: The Rush Memory and Aging Project: study design and baseline characteristics of the study cohort. Neuroepidemiology 2005;25: 163-175.

-9 Folstein MF, Folstein SE, McHugh PR: 'Minimental state'. A practical method for grading the cognitive state of patients for the clinician. J Psychiatr Res 1975;12:189-198.

10 Wilson RS, Barnes LL, Krueger KR, Hoganson G, Bienias JL, Bennett DA: Early and late life cognitive activity and cognitive systems in old age. J Int Neuropsychol Soc 2005; 11 : 400-407.

11 Bennett DA, Wilson RS, Schneider JA, et al: Natural history of mild cognitive impairment in older persons. Neurology 2002;59: 198-205.
12 McKhann G, Drachman D, Folstein M Katzman R, Price D, Stadlan EM: Clinical diagnosis of Alzheimer's disease: report of the NINCDS-ADRDA Work Group under the auspices of Department of Health and Human Services Task Force on Alzheimer's Disease. Neurology 1984;34:939-944.

13 Kim J, Sapienza CM: Implications of expiratory muscle strength training for rehabilitation of the elderly: tutorial. J Rehabil Res Dev 2005;42:211-224.

14 Enright PL, Kronmal RA, Manolio TA, Schenker MB, Hyatt RE: Respiratory muscle strength in the elderly. Correlates and reference values. Cardiovascular Health Study Research Group. Am J Respir Crit Care Med 1994;149:430-438.

15 Buchman AS, Wilson RS, Boyle PA, Bienias JL, Bennett DA: Motor function and mortality in older persons. J Am Geriatr Soc 2007; 55:11-19.

16 Wang CY, Olson SL, Protas EJ: Test-retest strength reliability: hand-held dynamometry in community-dwelling elderly fallers. Arch Phys Med Rehabil 2002;83:811-815.

17 McPhillips JB, Pellettera KM, Barrett-Connor E, Wingard DL, Criqui MH: Exercise patterns in a population of older adults. Am J Prev Med 1989;5:65-72.

18 Boyle PA, Wilson RS, Aggarwal NT, et al: Parkinsonian signs in subjects with mild cognitive impairment. Neurology 2005;65: 1901-1906.

19 Iqbal A, Schloss S, George D, Isonaka S: Worldwide guidelines for chronic obstructive pulmonary disease: a comparison of diagnosis and treatment recommendations. Respirology 2002;7:233-239.

20 Wilson RS, Beckett LA, Barnes LL, et al: Individual differences in rates of change in cognitive abilities of older persons. Psychol Aging 2002;17:179-193.

21 Laird NM, Ware JH: Random-effects models for longitudinal data. Biometrics 1982;38: 963-974.

22 Collett D: Modelling Survival Data in Medical Research, ed 2. Boca Raton, Chapman \& Hall, 2003.

23 SAS: SAS/STAT ${ }^{\circledR}$ User's Guide, Version 8, ed 8. Cary, SAS Institute, 2000.

24 Enright PL, McBurnie MA, Bittner V, et al: Cardiovascular Health Study. The 6-min walk test: a quick measure of functional status in elderly adults. Chest 2003;123:387393.

-25 Simpson CF, Punjabi NM, Wolfenden L, Shardell M, Shade DM, Fried LP: Relationship between lung function and physical performance in disabled older women. J Gerontol A Biol Sci Med Sci 2005;60:350354 .
26 Cook NR, Albert MS, Berkman LF, Blazer D, Taylor JO, Hennekens $\mathrm{CH}$ : Interrelationships of peak expiratory flow rate with physical and cognitive function in the elderly: MacArthur Foundation Study of Aging. J Gerontol A Biol Sci Med Sci 1995;50:M317M323.

27 Monso E, Fiz JM, Izquierdo J, et al: Quality of life in severe chronic obstructive pulmonary disease: correlation with lung and muscle function. Respir Med 1998;92:221-227.

28 Gosselink R, Troosters T, Decramer M: Peripheral muscle weakness contributes to exercise limitation in COPD. Am J Respir Crit Care Med 1996;153:976-980.

29 Troosters T, Gosselink R, Decramer M: Chronic obstructive pulmonary disease and chronic heart failure: two muscle diseases? J Cardiopulm Rehabil 2004;24:137-145.

>30 Hui DS, Joynt GM, Wong KT, et al: Impact of severe acute respiratory syndrome (SARS) on pulmonary function, functional capacity and quality of life in a cohort of survivors. Thorax 2005;60:401-409.

-31 Dishman RK, Berthoud HR, Booth FW, et al: Neurobiology of exercise. Obesity 2006;14: 345-356.

32 Rantanen T, Guralnik JM, Sakari-Rantala R, et al: Disability, physical activity, and muscle strength in older women: the Women's Health and Aging Study. Arch Phys Med Rehabil 1999;80:130-135.

-33 Fiatarone MA, Marks EC, Ryan ND, Meredith CN, Lipsitz LA, Evans WJ: High-intensity strength training in nonagenarians. Effects on skeletal muscle. JAMA 1990;263: 3029-3034.

>34 Keysor JJ, Jette AM: Have we oversold the benefit of late-life exercise? J Gerontol A Biol Sci Med Sci 2001;56:M412-M423.

35 Warburton DE, Nicol CW, Bredin SS: Health benefits of physical activity: the evidence. Can Med Assoc J 2006;174:801-809.

>36 Meyer FJ, Lossnitzer D, Kristen AV, et al: Respiratory muscle dysfunction in idiopathic pulmonary arterial hypertension. Eur Respir J 2005;25:125-130.

-37 Ferrucci L, Bandinelli S, Benvenuti E, et al: Subsystems contributing to the decline in ability to walk: bridging the gap between epidemiology and geriatric practice in the InCHIANTI study. J Am Geriatr Soc 2000;48: 1618-1625. 